\title{
Vibration Signature of Normal and Notched Tooth Gear Pump
}

\author{
Ahmed H. Osman, Assist. Prof., \\ Mohamed H. Gobran, Associate Prof., \\ Farouk F. Mahmoud, Master Student, \\ Mechanical Power Engineering Department, \\ Faculty of Engineering, Zagazig University, Zagazig, Egypt
}

Doi: 10.19044/esj.2019.v15n15p64 URL:http://dx.doi.org/10.19044/esj.2019.v15n15p64

\begin{abstract}
Gear pumps are critical devices in many industrial applications. An unexpected failure of the gear pump may cause significant effect on its performance. Consequently, there will be economic losses. As a result of that, fault diagnosis in gears has been the subject of intensive research. Vibration analysis has been used as an effective tool in machines diagnosis and in machinery maintenance decisions. As a rule, an increased vibration level is a warning form before failure or breakdown. By measuring and analyzing the gear pump vibration, it is possible to determine both the nature and severity of the defect, and hence predict the machine's failure. The vibration signal of a gear pump carries the signature of the fault in the gears, and early fault detection of the gear pump is possible by analyzing the vibration signal using different signal processing techniques. This paper presents, experimentally, the external gear pump signature for normal and faulty gear pumps at different rotational speeds $(1080,1200$, and 1439rpm). The considered faults herein are two different notches on one of the pump teeth - small notch and large notch. The paper concludes that features of the vibration are different with the notch shape and the rotational speed. The amplitude of vibration increases by increasing both rotational speed and notch size.
\end{abstract}

Keywords: External Gear Pump, Vibration Analysis, Fast Fourier Transform, fault diagnosis

\section{Introduction}

Gear pumps are fixed positive displacement machine. A gear pump uses the meshing of gears to pressurize fluid by displacement. They are one of the most common types of pumps for fluid power applications. Gear pump type is widely used in chemical installations to pump highly viscosity fluids. 
It is also used as lubrication pump in machine tools, in fluid power transfer units, and as oil pumps in engines. Therefore, there are two main variations; external gear pumps which use two external spur gears and internal gear pumps which make use of an external and an internal spur gear. Small external gear pumps usually operate at 1750 or $3450 \mathrm{rpm}$, while larger ones operate at speeds up to 640rpm.

The pump is susceptible to encounter many physical problems such as pitting, scuffing, spalling, cracking, wear, and excessive clearance. These problems may cause rotor imbalance and consequently high vibration level. Then the vibration analysis is an effective tool for the gear pump condition monitoring. The approaches of gear vibration analysis are mainly subdivided into three categories according to analysis domains; time domain, frequency domain, and time-frequency domain.

Gupta et al. (2006) stated that lubrication of gears has shown a beneficial effect. In this case, vibration levels are smaller which has resulted in smaller RMS, crest factor, and Kurtosis values. The effect of defect in gear was not that significant on these parameters. The spectra of the velocity and acceleration signals contained rotational speed and its higher harmonics. A close interaction exists between the system natural frequencies and the gear mesh frequency. The natural frequency in close vicinity of the gear mesh frequency showed a dominant peak. Significant sidebands exist in all the spectra. The spectra of the acceleration signals showed predominantly the frequencies generated by the bearings which interact with system natural frequencies in the overlapping frequency range.

Spectra Quest Inc. (2007) conducted an experimental work for Gearbox Dynamics Simulation. This is to investigate the gear fault diagnosis of two-stage parallel gear transmission with and without load. Three faults were made: small teeth notch, large teeth notch, and missing teeth. When the load is applied (in both healthy and faulty gear), higher amplitude of vibration arises compared with the no load condition. Chipped tooth and missing tooth generate an impact in every revolution. Therefore, sidebands around the meshing frequencies appear. The frequency interval is the input shaft speed. The severity level of the chip affects the sideband amplitudes. In some cases, the amplitudes of the sidebands are even higher than that of the meshing frequency component, which is quite different from the behavior of a healthy gear.

Lei You et al. (2011) presented a new fault diagnosis system of rotating machinery vibration signal to improve the precision of testing vibration signal. They used an advanced PXI test platform, adopting 16 bits resolution A/D device and FPGA technology in the design. The conditions for further realization of detail analysis of time-frequency domain were also provided. The system can monitor vibration acceleration and velocity signal, 
thus making accurate judgment on mechanical fault. Compared with the existed fault diagnosis systems, the system mentioned in this paper can achieve higher accuracy. In addition, it can also improve accurate diagnostic capability and dramatic increase.

Diwakar et al. (2012) introduced an experimental study which has been conducted on motor with Gearbox measured quantities such as frequency and amplitudes. The peaks are present at sub-harmonics and multiples of frequencies. The cause of presenting the sub-harmonics and multiples of frequencies is due to the presence of fault in the Gearbox.

Renata (2013) presented and discussed two methods of separating between signals generated by different sources of faults. These methods are liftering in the frequency domain and dephase. The methods are compared using both simulated signals and real data. For separation of discrete frequencies noise, the method of dephase is superior relative to frequency liftering. In the case of synchronous shaft speeds, a modified flow of algorithms was suggested for efficient realization of the dephase process. A new method for isolation of transmission path effects, adaptive clutter separation (ACS), was proposed. The method was compared to cepstrum liftering. In the frequency domain, cepstrum liftering was found superior to ACS. On the other domain, ACS provided better results. It seems that ACS is more effective for prewhitening while cepstrum liftering is preferable for estimation of transmission path effects.

Rishi Kumar Sharma et al. (2014) observed the FFT spectrum of healthy gear and gear with Breakage fault; they concluded that the effect of gear tooth breakage appears in frequency domain vibration signal as Sidebands of fundamental frequency. In addition, the amplitudes of harmonics in case of gearbox with breakage fault are more than the amplitude of harmonics of healthy gearbox. The presence of tooth breakage fault in any gearbox gives rise to peaks and generates sidebands. From FFT technique, it can be predicted that the gearbox has some fault, but the severity cannot be determined.

Alexander et al. (2014), applied a combined analytical and empirical approach to study the effect of different faults on the vibrational pattern. Dynamic model generated signatures are used to verify experimental findings. Moreover, discrepancies between simulated and actual results, combined with understanding of the assumptions and omissions of the model clarify and explaining the experimental results. A spur gear transmission setup was used for experiments, along with an electric AC motor and a friction belt-loading device. The experimental runs were conducted at varying speed settings. There are two types of faults, a tooth face fault and a tooth root fault. 
Dadon et al. (2015) introduced an approach of measuring the vibration pattern. This approach based on empirical observations, derived from actual measurements of vibration patterns, was examined. In the study, a simple spur gear transmission system is modelled and analyzed. In the first stage, a nonlinear dynamic model of a spur gear transmission was developed. In the second stage, an analytical method was proposed to estimate the gear mesh stiffness as a function of mesh angle in the case of both healthy and faulty gears. The dynamic response of the system was computed by applying the gear mesh stiffness derived from the analytical model. The model was applied to simulate the theoretical vibration response in healthy and spalled tooth gearwheels. The dynamic responses derived from the model were compared to and verified with experimental vibration data. The experimental phase included varying the load and speed settings, allowing the examination of the fault effect on the vibration signature under different test conditions.

Gawali S.N. (2015) used vibration analysis technique to detect faults in gearbox system. In vibration analysis, technique gear faults are detected based on Time-Frequency analysis with the help of MATLAB software. Various types of gear defects can be artificially induced on gear tooth such as missing tooth, one corner defect, two corner defect, inadequate lubrication, etc. This can be done by comparing the result of signals of healthy condition gear with defective condition through the FFT analyzer. Analysis is carried out with graphs of high frequency vibrations. With the help of MATLAB software, validation of this data was successfully carried out. This paper has examined the Gear fault detection using feature extraction parameters time, frequency domain parameter, and vibration monitoring

Ajanalkar Sagar Shivputra et al. (2016) experimental setup is prepared and the vibration analysis is carried out at different speeds to analyze the behavior of time waveform and FFT spectrum for three different fault conditions. The gear pair of Bajaj Pulsar gearbox is taken, and the faults are created on pinion and the vibration behavior is observed. The three fault conditions are single tooth damaged pinion, double teeth damaged pinion, and worn pinion. In addition, some of the numerical techniques like RMS value and variance are used for comparing the behavior of faulty pinion with respect to healthy pinion.

\section{Pump Description}

The studied healthy model is an external gear pump as shown in Figure 1. It is a simple one-stage spur gear system of Standard profile spur gear pair. Each gear has a module of $2.5 \mathrm{~mm}, 12$ number of teeth, and a width of about $19 \mathrm{~mm}$. The pinion is seated on the "In" shaft. The transmission reduces the speed of the "Out" shaft containing the driven gear and the loading device. There are two ball bearings and each support both shafts. 
There is one meshing tooth. Two deteriorated of faulty models were examined; small and large notches in a single tooth. Figure 1 through 3 illustrate healthy, small notch and large notch gears respectively. Figures 2 and 3 give the configuration of both small and large notch tooth.

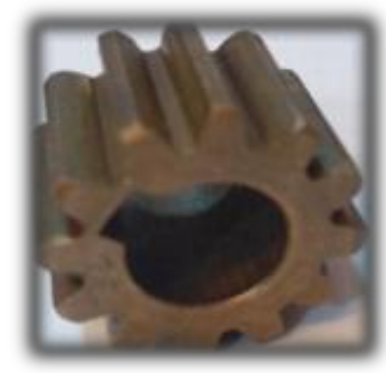

Figure 1. Healthy teeth

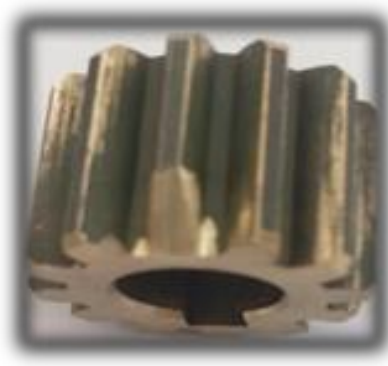

Figure 2. Small tooth notch

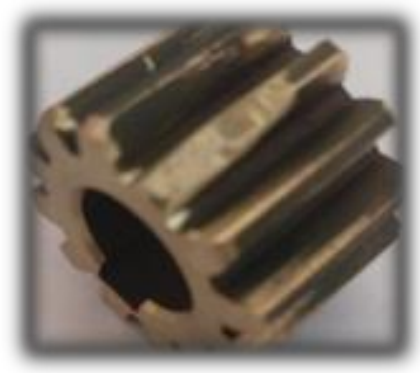

Figure 3. Large tooth notch

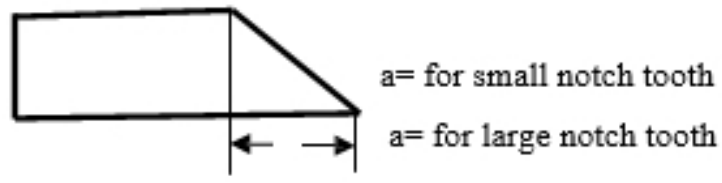

Figure 4. Shape of tooth notch

\section{Experimental Test Rig \\ Test Rig Description}

Figure 5 shows diagrammatic sketch of the experimental hydraulic test rig for measurements which is used in this study. The test rig consists of $36 \times 11.5 \times 17 \mathrm{~cm}$ oil tank (1), oil filter (2), tested gear pump (3), $0.5 \mathrm{HP}$ electric motor running at $1500 \mathrm{rpm}(4)$, pressure relief valve (5), manual operated $4 / 3$ directional control valve (6), and double acting hydraulic cylinder (7). The experiment was carried out at a neutral position of a closed center directional control valve (no flow to or from the cylinder). Then the system operates at a pressure equals the relief valve setting pressure (50bar). 


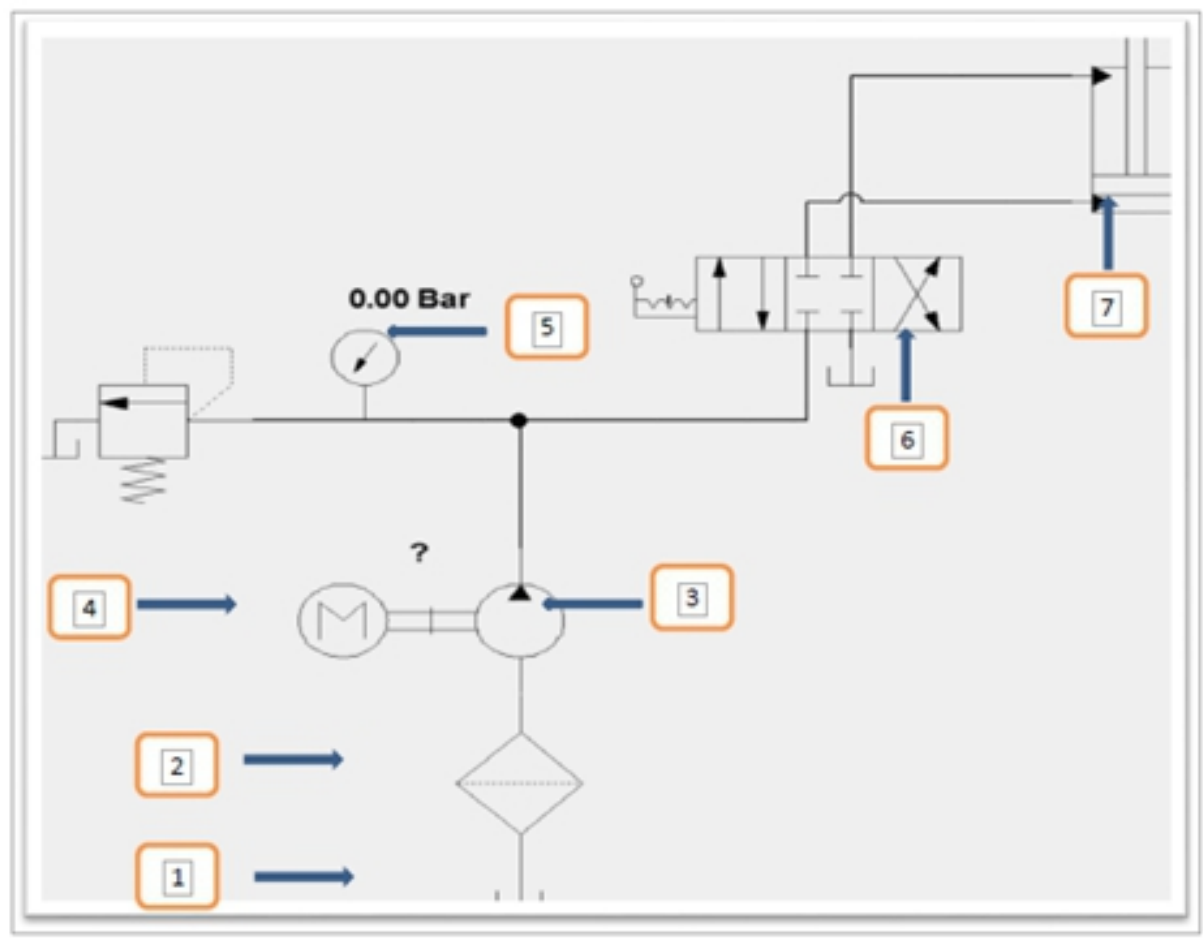

Figure 5. Hydraulic circuit

\section{Instrumentation Apparatus}

The system pressure is measured by pressure Gauge; it has a range from 0.0 to 200 bar. The vibration is measured by Dytran 3093B. Triaxial Integrated Electronic Piezoelectric (IEPE) Accelerometer, Table 1 gives some specifications of accelerometer. The speed of an electric motor speed is changed by using frequency converter modulator.

Table 1. Specifications of sensor

\begin{tabular}{|l|l|}
\hline Brand & Dytran \\
\hline Accelerometer Type & Triaxial \\
\hline Sensitivity & $10 \mathrm{mV} / \mathrm{m} / \mathrm{s}^{2}$ \\
\hline Frequency Response & 0.6 to $5000 \mathrm{~Hz}$ \\
\hline Frequency Response Gain & $\pm 10 \mathrm{~dB}$ \\
\hline Temperature Range & -51 to $+121^{\circ} \mathrm{C}$ \\
\hline
\end{tabular}

\section{Data Acquisition System (DAS)}

A typical DAS consists of the following components (Ahmed, 2013):

1. Signal Conditioning where Charge amplifier is used to convert and amplify AC signal from sensor to DC signal.

2. A/D Card; the function of this hardware is to convert analog signals into digital signals. 
3. Screw terminal, to connect wires.

4. PC computer.

5. Software (Test point program).

Figure 6 illustrates the component of data Acquisition System:

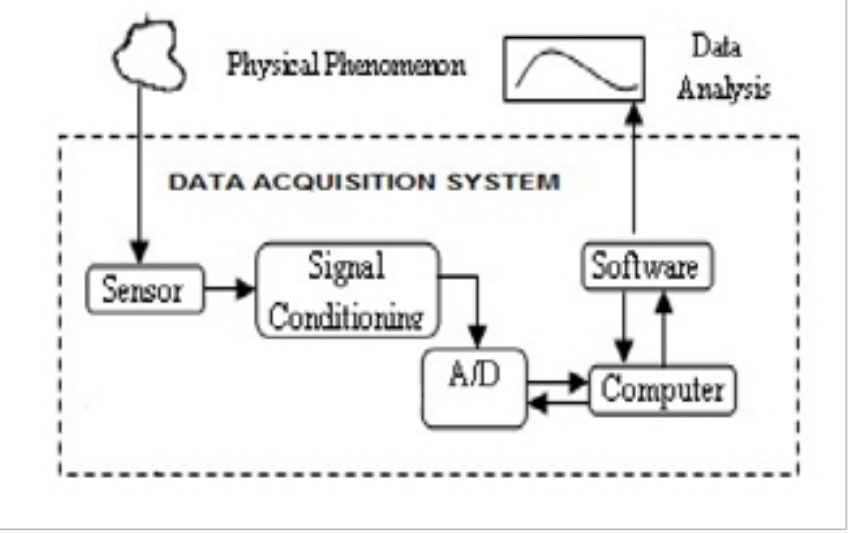

Figure 6. Data Acquisition System

\section{Test Procedure}

1. Physical variable is a vibration on gear pump.

2. Vibration sensor model (DYTRAN 30938) has 3 electric cables that sends AC signal.

3. Charge amplifier is used to convert and amplify AC signal from sensor to DC signal.

4. Screw terminal is used to connect the electrical cables.

5. Analog signals are converted to digital signals by A/D Card.

6. Data cable is responsible for transporting digital signal from $\mathrm{A} / \mathrm{D}$ card to personal computer.

7. Personal computer is a device use to see the software program.

8. Software program is used to appear and record data through $\mathrm{A} / \mathrm{D}$ card to PC screen by using TESTPOINT Program.

\section{Experimental Results}

The vibration is measured at different pump speeds of 1080, 1200, and 1439RPM by using the accelerometer for both healthy and faulty pump (normal, small notch, and large notch). The motor speed is adjusted by using the frequency modulator. Table 2 gives the corresponding frequency for each running speed. A computer program, based on MATLAB, is constructed to read and draw the test results. Thus, the experiment runs using the following steps: 
1. Start/turn on the electric switch to run the system.

2. Set frequency modulator to adjust the running speed $(1080,1200$, and1439 RPM).

3. The "Test point" program is activated to record the test data.

4. The data recorded by the "Test Point" program is sent to the MATLAB program to plot and analyze it.

Table 2. The rotating speed and frequency from frequency modulator

\begin{tabular}{|c|c|}
\hline Rotating speed, RPM & Frequency, $\mathrm{Hz}$ \\
\hline 1080 & 36.5 \\
\hline 1200 & 40.5 \\
\hline 1439 & 48.5 \\
\hline
\end{tabular}

\section{Feature Extraction}

Figure 7 through to 9 shows the vibration behavior in frequency domain (amplitude of acceleration in $\mathrm{mm} / \mathrm{s}^{2}$ with the frequency in HZ). These figures illustrate the vibration behavior for small and large notch models compared with normal model for variable running speeds of 1080, 1200 and 1439RPM, respectively. These figures demonstrate that for all running speeds, the amplitude of vibration of large notch is higher than that of small notch and normal condition. Also, for all models, the amplitude of vibration increases as the running speed increases.
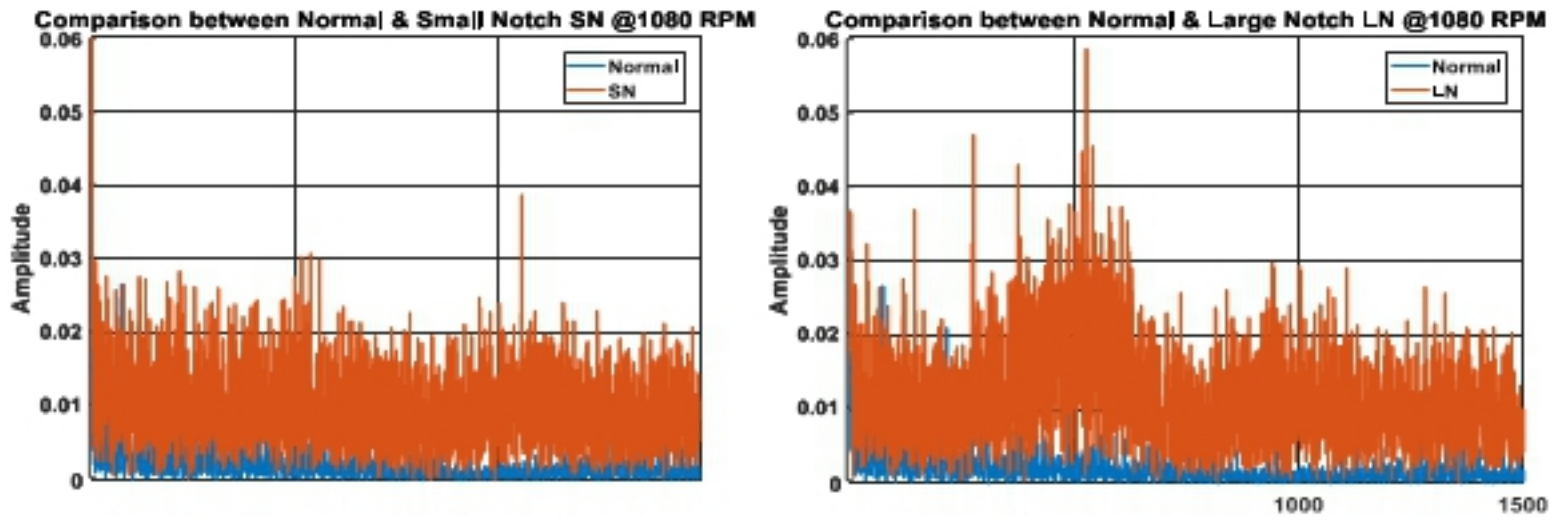

Figure 7. Comparisons between normal, small notch and large notch at speed 1080RPM 

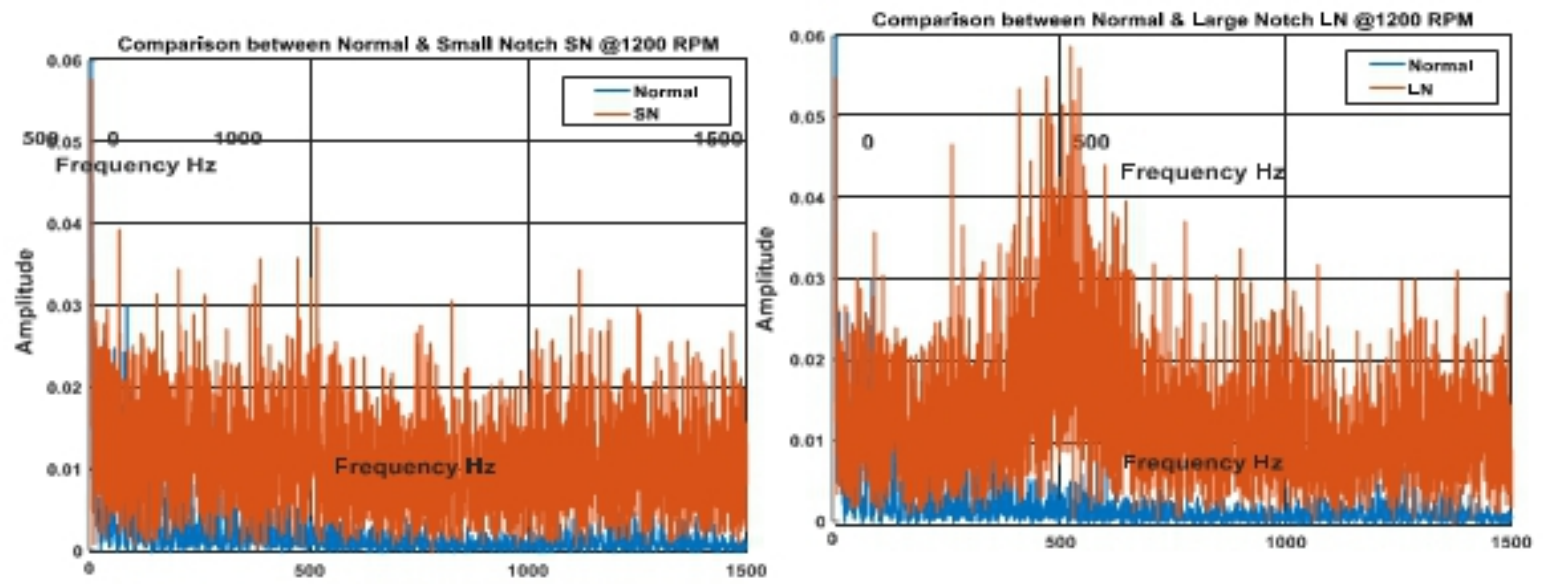

Figure 8. Comparisons between normal, small notch and large notch at speed 1200RPM
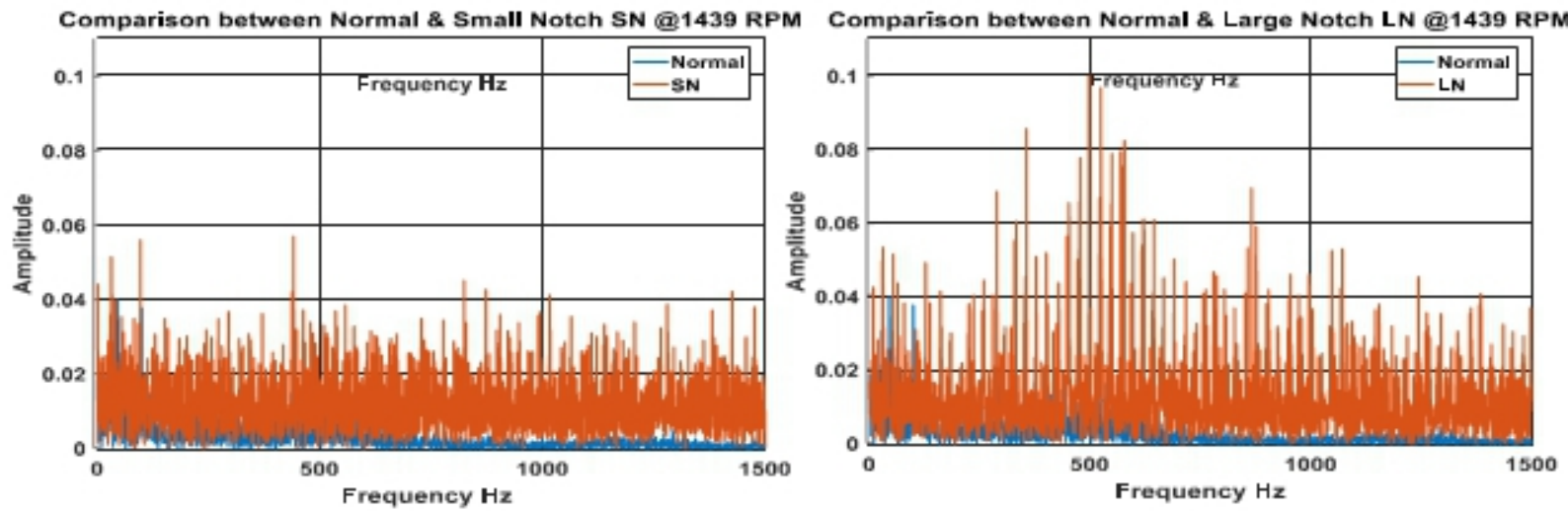

Figure 9. Comparisons between normal, small notch and large notch at speed 1439RPM

\section{Envelope Method}

This method Compute and plot the upper and lower RMS envelopes of the signal of a feature extraction for vibration level with a length of 100 samples. Figure 10 shows the envelope of normal, small notch and large notch condition at all speed $(1080,1200$, and1439). The figure illustrates the clarity feature between all speeds at all faults. The amplitude at large notch is bigger than the small notch and normal condition. 

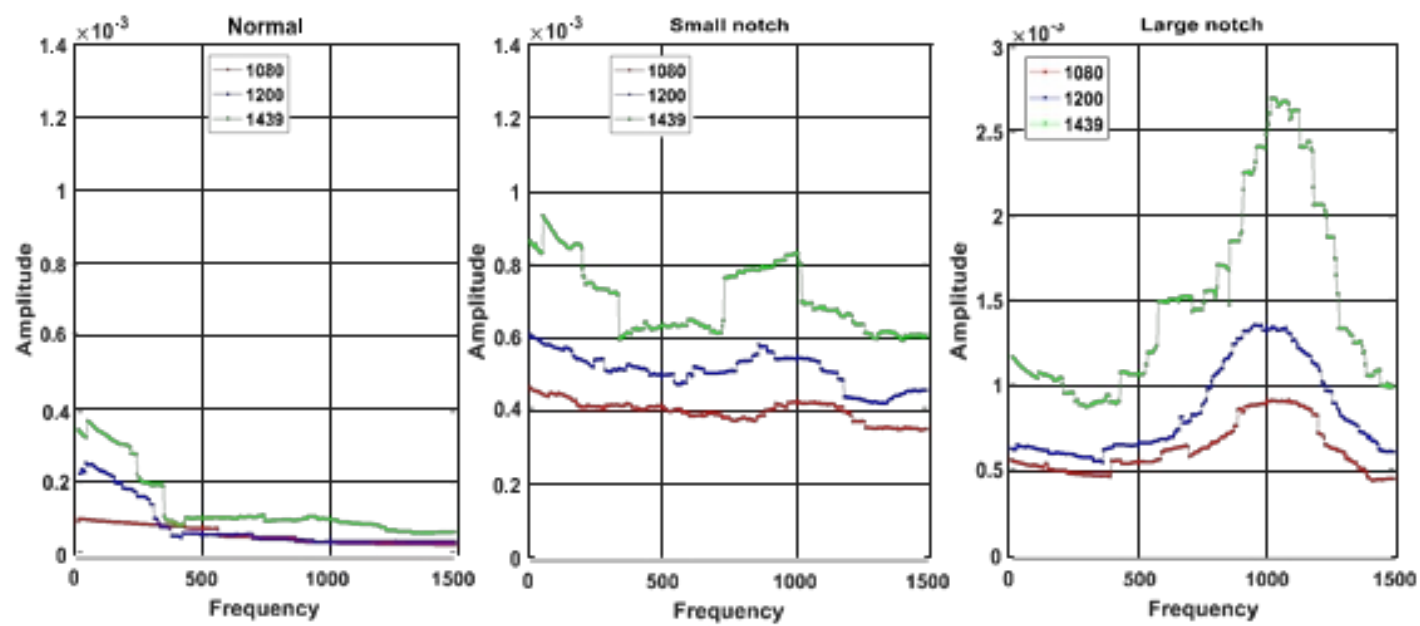

Figure 10. Envelope between normal, small notch and large notch condition at all speeds

$(1080,1200$ and 1439)

\section{Integration of Fast Fourier Transform}

Figure 10 shows comparison between Normal, Small and Large Notch for all speeds by using relation between the integration of FFT $\left(f(t) / \omega^{2}\right)$ and RPM as shown also in the table. Figure 11 also shows that the large notch has a greater value than the small notch and the normal condition. Table 3 shows the numerical values of the feature.

Table 3. The Numerical values for the feature at all faults and RPM

\begin{tabular}{|r|r|r|r|r|}
\hline & $1080 \mathrm{RPM}$ & $1200 \mathrm{RPM}$ & \multicolumn{1}{|c|}{$1439 \mathrm{RPM}$} \\
\hline Normal & $4.71 \mathrm{E}-05$ & $4.01 \mathrm{E}-05$ & $4.49 \mathrm{E}-05$ & \\
\hline Small Notch & $2.64 \mathrm{E}-04$ & $2.37 \mathrm{E}-04$ & & $1.76 \mathrm{E}-04$ \\
\hline Large Notch & $2.93 \mathrm{E}-04$ & $2.68 \mathrm{E}-04$ & & $1.79 \mathrm{E}-04$ \\
\hline
\end{tabular}

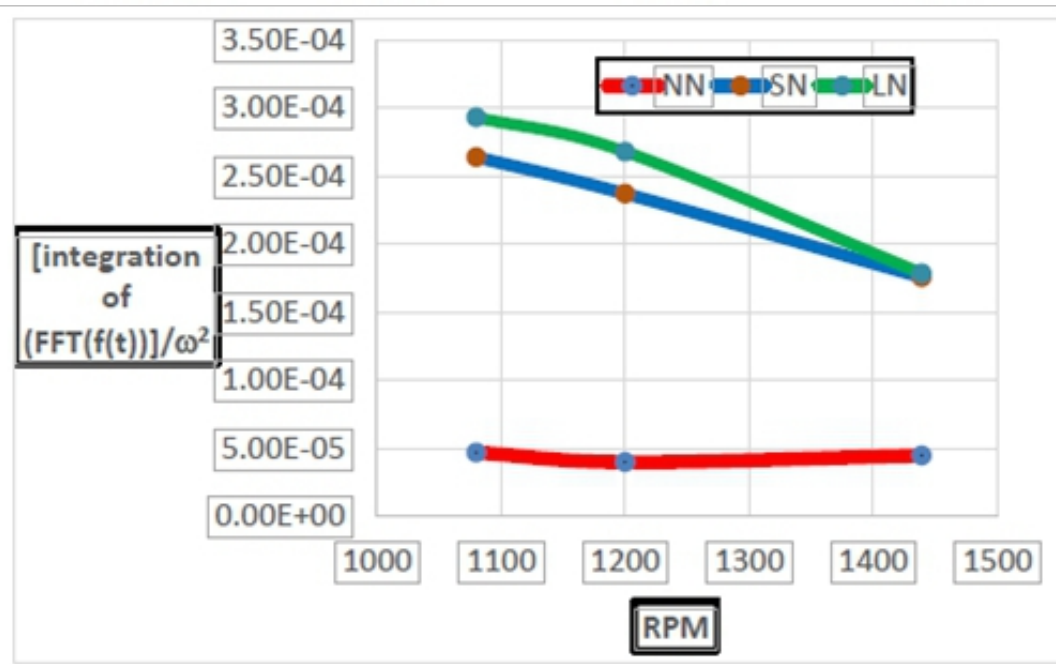

Figure 11. Comparison between Normal, Small, and Large Notch at all speed $(1080,1200$, and 1439) 


\section{Conclusion}

Experiments were conducted on gear pump to investigate the effects of the tooth notch as a fault in the vibration features. Two notches were investigated; one is a "small notch" while the other is a "large one". Three techniques were used to explain the effect of these faults on the vibration level at different pump rotational speeds (1080, 1200, and 1439rpm). These techniques are: Feature Extraction, Envelope, and Fast Fourier transformation.

From the experimental results, the following conclusions were drawn:

- More speed result in more amplitude of vibration for all conditions (healthy and deteriorated).

- The amplitude of vibration at large notch is larger than the small notch for all tested speeds.

- The vibration level of deteriorated models is higher than that of healthy model for all speeds.

- Envelope technique illustrates the magnitude of RMS of 100 samples. The RMS magnitude increases with the level of notch for all speeds. For 1439rpm, the maximum amplitudes occur nearly at $1000 \mathrm{~Hz}$ $\left(2.7 * 10^{-3}, 1.4 * 10^{-3}\right.$ and $0.8 * 10^{-3}$ for 1439,1200 , and 1080 , respectively)

- The integration of FFT $\left[f(\mathrm{t}) / \omega^{2}\right]$ is the best method for comparison between Normal, Small, and Large Notch for all speeds.

\section{References:}

1. Ahmed, H.O. \& Magdy, M.M. (2013). "Setting up a Data Acquisition System for Spark Engine", 1st Annual International Interdisciplinary Conference, AIIC 24-26 April, Azores, Portugal.

2. Alexander Bliznyuk, et al. (2011). "Gear Diagnostics - Fault Type characteristics" Laboratory for Health, Department of Mechanical Engineering, Ben-Gurion University of the Negev, Beer- Sheva, Israel open-access article.

3. Ajanalkar Sagar Shivputra, et al. (2016). "Gear Fault Identification by using Vibration Analysis " 247| MIT College of Engineering, Pune, India, AMET 2016, INPRESSCO IJCET Special Issue-4 (March 2016).

4. Dadon et al. (2015). "Towards a reliable non-linear dynamic model of damaged gear transmission", The British Institute of Non-Destructive Testing.

5. Gawali, S. N. (2015). "Condition Monitoring Technique to Investigate Gear Fault with Artificially Induced Gear Crack by using Vibration 
Analysis "International Engineering Research Journal Page No 20732078.

6. Gupta, P., et al. (2006). "Experimental Investigation of Vibrations in In a Geared System", Proceeding of International Conference on Resource Utilization and Intelligent Systems At: Erode, T.N., India, Volume: pp. 1-6

7. Rishi Kumar Sharma, et al. (2014). "Analysis of Spur Gear Faults using Frequency Domain Technique" international Journal of Engineering Sciences \& Research Technology [258-262].

8. Lei You et al, 2011, "Fault diagnosis system of rotating machinery vibration signal" Elsevier ltd, 1877 - 7058, pp671 - 675

9. Renata Klein (2013), "Comparison of Methods for Separating Excitation Sources in Rotating Machinery", R.K. Diagnostics Gilon, D.N. Misgav, P.O. Box 101, 20103, Israel

10. S. N. Gawali, (2015) "Condition Monitoring Technique to Investigate Gear Fault with Artificially Induced Gear Crack by using Vibration Analysis "International Engineering Research Journal pp 2073-2078

11. Spectra Quest, Inc, August (2007), "Vibration Signature of Defected Gear Tooth Using Spectral Analysis", www.spectraquest.com, 8201 Hermitage Road, Richmond, VA 23228 